\title{
Efficacy of intra-articular hypertonic dextrose prolotherapy versus normal saline for knee osteoarthritis: a protocol for a triple-blinded randomized controlled trial
}

Regina Wing Shan $\mathrm{Sit}^{1 *} \mathbb{D}$, Ricky Wing Keung Wu', Kenneth Dean Reeves², David Rabago ${ }^{3}$,

Dicken Cheong Chun Chan ${ }^{1}$, Benjamin Hon Kei Yip ${ }^{1}$, Vincent Chi Ho Chung ${ }^{1}$ and Samuel Yeung Shan Wong ${ }^{1}$

\begin{abstract}
Background: Knee Osteoarthritis (KOA) is a very common condition with prevalence rising with age. It is a major contributor to global disability and has a large socioeconomic burden worldwide. Conservative therapies have marginal effectiveness, and surgery is reserved for severe symptomatic KOA. Dextrose Prolotherapy (DPT) is an evidence-based injection-based therapy for chronic musculoskeletal conditions including KOA. The standard "whole joint" injection method includes intra-articular injection and multiple extra-articular injections at soft tissue bony attachments. The procedure is painful and requires intensive procedural training often unavailable in conventional medical education, which potentially limits access. Intra-articular injection offers the possibility of a less painful, more accessible treatment. The aim of this project is to assess the clinical efficacy of intra-articular injection of DPT versus normal saline (NS) for KOA.
\end{abstract}

Method: Seventy-six participants with KOA will be recruited from the community. We will conduct a single center, parallel group, superiority randomized controlled trial comparing DPT and NS injections, with blinding of physician, participants, outcome assessors and statisticians. Each group will receive injections at week 0, 4, 8 and 16. The primary outcome will be the Western Ontario McMaster University Osteoarthritis Index pain scale (WOMAC), and secondary outcomes include WOMAC composite score, the WOMAC function and stiffness subscale, the Visual Analogue Score of pain, objective physical function tests (the $30 \mathrm{~s}$ chair stand, 40- $\mathrm{m}$ fast paced walk test, the Timed up and go test) and the EuroQol-5D (EQ-5D). All outcomes will be evaluated at baseline, and 16, 26 and 52 weeks. All analyses will be conducted on an intention-to-treat basis using linear mixed regression models.

Discussion: This paper presents the rationale, design, method and operational aspects of the trial. The findings will determine whether IA DPT, an inexpensive and simple injection, is a safe and effective non-surgical option for KOA. The results can be translated directly to clinical practice, with potentially substantial impact to patient care.

Trial registration: The trial (ChiCTR-IPC-15006617) is registered under Chinese Clinical Trials Registry on $17^{\text {th }}$ June 2015.

Keywords: Dextrose prolotherapy, Normal saline, Randomized controlled trial, Knee osteoarthritis, Study protocol

\footnotetext{
*Correspondence: reginasit@cuhk.edu.hk

'Jockey Club School of Public Health and Primary Care, The Chinese

University of Hong Kong, New Territories, Hong Kong

Full list of author information is available at the end of the article
}

(c) The Author(s). 2018 Open Access This article is distributed under the terms of the Creative Commons Attribution 4.0 International License (http://creativecommons.org/licenses/by/4.0/), which permits unrestricted use, distribution, and reproduction in any medium, provided you give appropriate credit to the original author(s) and the source, provide a link to the Creative Commons license, and indicate if changes were made. The Creative Commons Public Domain Dedication waiver (http://creativecommons.org/publicdomain/zero/1.0/) applies to the data made available in this article, unless otherwise stated. 


\section{Background}

Knee osteoarthritis (KOA) is a common chronic arthritis leading to joint pain and disability worldwide [1]. KOA is age-related [2]; by age 65 , around $30 \%$ of the population has osteoarthritis [3, 4]. KOA is an expensive disease with significant socioeconomic burden due to its high prevalence, worker absenteeism and costly health care utilization $[5,6]$. While exercise and weight reduction are effective in KOA, factors such as fatigue, accessibility and the arthritis itself have been identified as barriers for the actual participation [7]. Other conservative therapies such as physiotherapy, oral analgesic medications and complementary therapies such as acupuncture and herbal treatment have marginal effectiveness [8-11]. Total knee replacement (TKR) for advanced KOA is effective but costly [12].Thus, a safe and effective treatment option that complements the current conservative therapy remains a top priority in clinical practice and research $[13,14]$.

Pain and functional impairment in KOA are associated with a multifactorial set of degenerative intraarticular cartilage, bone and synovial knee structures, in addition to a complex interaction between genetic, biochemical, biomechanical, psychosocial, and other factors such as neurogenic inflammation and central pain sensitization [15-17]. The heterogeneous pain mechanisms in KOA may explain the variable responses to different therapies, and the search for effective non-surgical treatment for KOA has been challenging.

Dextrose prolotherapy (DPT) is an injection-based therapy for chronic musculoskeletal pain conditions including KOA [18]. The mechanism of action is likely multifactorial, and is hypothesized to work through stimulation of fibroblast and vascular proliferation, dense collagen deposition, and cartilage growth [19]. Additionally, dextrose solutions may have potential sensorineural analgesic effects as suggested recently by the effect of epidural injection of dextrose in the treatment of chronic non-surgical low back pain [20]. DPT may therefore treat KOA by targeting structural dysfunction, reducing nociceptive drive and minimizing peripheral sensitization.

The standard injection method of DPT involves a whole joint injection, consists of single intraarticular joint injection and multiple extra-articular injections at soft tissue attachments [21]. Pain and functional improvement in KOA have been reported in randomized controlled trials [22, 23], systematic review [24, 25] and meta-analysis [26, 27]. However, the procedure is painful due to multiple skin punctures, and premedication with a centrally acting opioid analgesic is sometimes used. In addition, the extra-articular injection protocol requires additional intensive postgraduate training, the access to which may be limited, minimizing availability of DPT.

The beneficial effects of a protocol using serial intra-articular dextrose injections have been reported in a small number of studies, though the results are limited by small sample size, lack of control arm or modest study design [28-32]. The current project aims to conduct a rigorous randomized controlled trial (RCT) to evaluate the intra-articular approach of DPT for KOA.

The primary aim of this study is to assess the clinical efficacy of intra-articular DPT versus normal saline (NS) in terms of self-reported knee pain at 52 weeks. We hypothesize that DPT is superior to NS in pain reduction as assessed by the Western Ontario McMaster University Osteoarthritis Index (WOMAC), a guideline recommended validated self-reported outcome in KOA trials [33]. Our secondary aims are to assess the clinical efficacy of intra-articular DPT versus NS in terms of subjective and objective functional improvement and quality of life at 52 weeks, using the WOMAC functional scale, $30 \mathrm{~s}$ chair stand, $40 \mathrm{~m}$ fast paced walk test, the Timed up and go test and EuroQuol-5D. EuroQuol-5D [34, 35].

\section{Methods \\ Study Design}

The study is a 52-week, two-arm, parallel, superiority randomized controlled trial aimed to evaluate the comparative effectiveness of DPT versus NS for KOA. The study design follows the recommendation from the Osteoarthritis Research Society International (OARSI) [33], and the reporting will follow the Standard Protocol Items: Recommendations for Interventional Trials (SPIRIT) [36]. The study has been approved by the Joint Chinese University of Hong Kong - New Territories East Cluster Clinical Research Ethics Committee.(CREC no. 2014.059). This trial has been prospectively registered in the Chinese Clinical Trial Registry on $17^{\text {th }}$ June 2015. (Registration no. ChiCTR-IPC-15006617) The study workflow is summarized in Fig. 1.

\section{Eligibility}

Eligibility will be screened by a trained research assistant using a phone interview, and potential eligible participants will be invited to meet the principal investigator (a physician) at the study site for confirming eligibility based on the following eligibility criteria:

Inclusion criteria include: (1) age $\geq 45$ to $\leq 75$ years old; we set the upper age limit to ensure adequate potential for cartilage regeneration [37]; (2) diagnosis of primary knee OA based on clinical and radiological criteria as defined by the American Rheumatology Association 


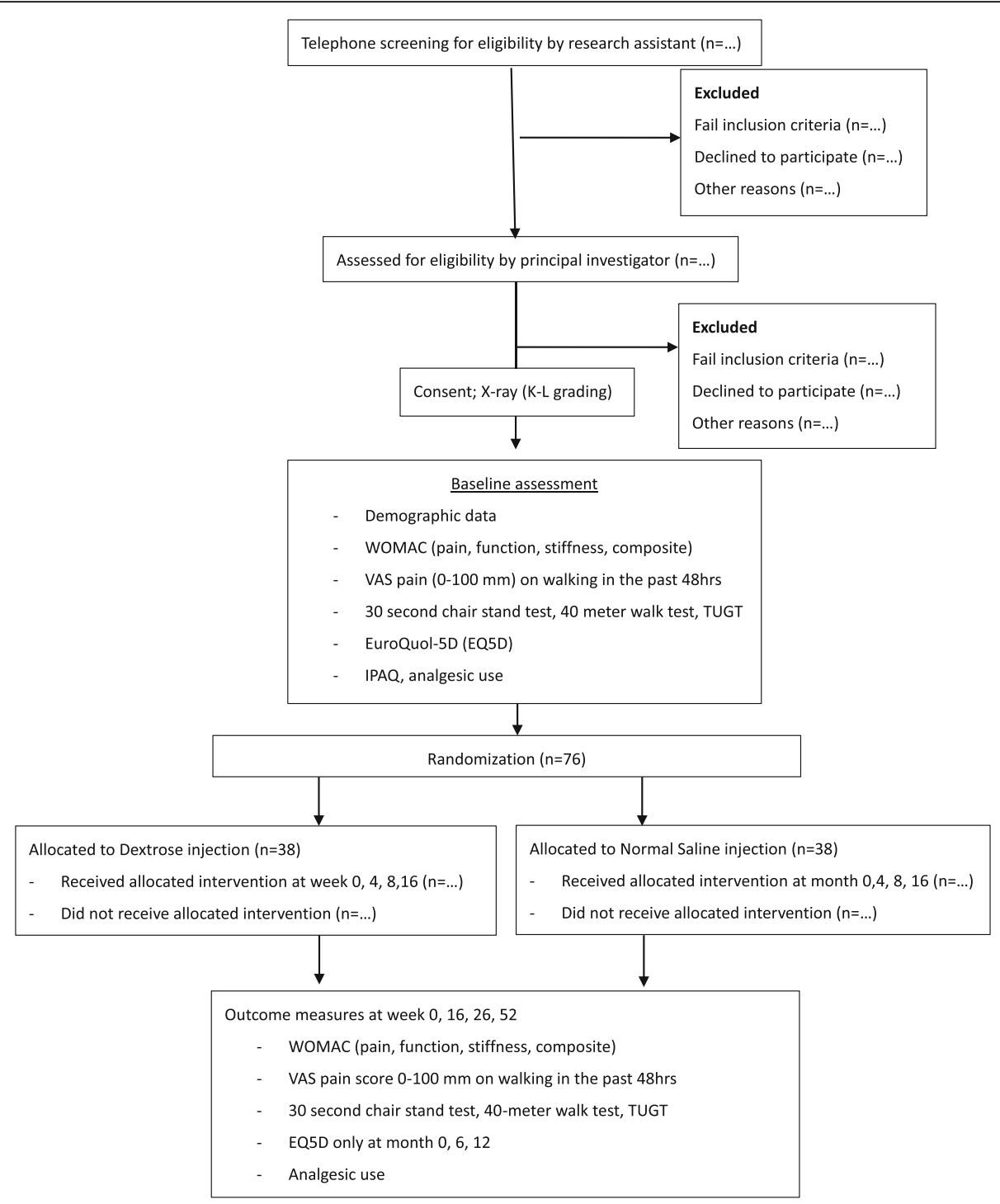

Fig. 1 Study flow chart

[38]; (3) moderate to severe knee pain for at least 3 months, defined as a score of $\geq 3$ (0-6 ordinal response scale) on the question "What is the average level of your left/right knee pain in the past 3 months?"; and (4) failure to achieve pain reduction to a score $<3(0-6$ ordinal response scale) after 6 months of usual care, such as weight reduction, exercise, physical therapy and pharmacological treatment.

Exclusion Criteria include (1) history of corn allergy [39, 40]; (2) previous knee replacement surgery on the referring knee; (3) pregnancy; (4) body mass index $(\mathrm{BMI}) \geq 35 \mathrm{~kg} / \mathrm{m}^{2}$; (5) patients on anticoagulant therapy; (6) prior knee injections within 3 months; (7) inflammatory or post-infectious knee arthritis, such as clinically diagnosed rheumatoid arthritis, gouty arthritis, psoriatic arthritis and septic arthritis; (8) significant effusion as defined by a ballotable patella; and (9) co-morbidity or lifestyle preventing participation in the study protocol.

\section{Recruitment and consent}

Participants will be recruited in the General Outpatients Clinics (GOPCs) in the New Territories East (NTE) region of Hong Kong. There are seven general outpatient/ family medicine clinics in NTE region that provide primary care services for the corresponding population, serving more than 1.2 million people. Subjects with KOA will be recruited via poster advertisement in GOPCs and direct physicians' referral. The study site is a teaching clinic operated by the Chinese University of Hong Kong. After confirming the eligibility of the participants, the principal investigator will take approximately 
15 min to describe the study goals, procedures, activities and possible alternatives, and answers all questions. Following this, interested prospective candidates will be given 7 days to consider the enrolment. The research assistant will then call the candidates for a second visit when written informed consent will be signed. After the enrolment, participants will receive a study identification number and undergo baseline data collection.

\section{Randomization and Allocation Concealment}

Blocked randomization in 1:1 ratio will be used to allocate patients to the two groups [41]. The sequence will be generated by the Random Allocation Software. Random sequence will be concealed using sequentially numbered, opaque sealed envelopes (SNOSE) procedure [42]. These SNOSE will be kept by a person not involved in the care or evaluation of patients, or in the data analysis. The investigator enrolling the patient will have no access to SNOSE. The treatment allocation process starts when the investigator calls the personnel keeping the SNOSE. The computer database is designed in such a way that treatment allocation cannot be changed after randomization. Each participant will receive the SNOSE and they will be asked to sign on it. The SNOSE will only be opened at 52-week.

\section{Blinding of participants and personnel}

Two registered nurses not involved in participant care will prepare the syringes with dextrose or NS identified only by study identification numbers. The syringes will be wrapped in aluminium foil to mask the solutions. The principal investigator and the study coordinator will therefore be blinded to group status of all subjects. The physician who conducts the injections will be blinded to the allocation group; he is also prohibited to communicate with the participants. Dextrose and saline solutions are odorless and identical in color and viscosity. Participants will be blinded to their group status, knowing only their randomization group number. To assess the success of participant blinding, participants will be asked to guess their group status at 12-month before unmasking, and data will be analyzed and interpreted using established procedure [43].

\section{Blinding in outcome assessment and data analysis process}

All data collection will be performed by trained research assistants blinded to the allocation status of the patients via face to face interviews. They will receive rigorous training in standardized data collection procedures. Data entry personnel external to the research team will be employed to perform data entry such that the statistician can analyze data without the need to refer to allocation information, thus ensure blinding.

\section{Baseline measurement}

Demographic data such as age, gender and BMI will be collected. Since objective functional outcome will be evaluated in this trial, the baseline physical activity status will be assessed using the Chinese version of International Physical Activity Questionnaire (IPAQ) [44]. Duration of knee pain and prior knee interventions such as weight reduction, knee exercise, physiotherapy, hyaluronic acid injection, corticosteroid injection or Traditional Chinese Medicine (TCM) etc. will be collected. All other co-morbidities will be documented as potential confounders. The severity of KOA will be graded by radiologist using the Kellgren-Lawrence Grading [45].

\section{Interventions}

Following sterile preparation and injection of $1 \mathrm{ml} 1 \%$ xylocaine as local anesthetic blebs, participants will be injected under ultrasound guidance with 25-gauge needle directed to the suprapatellar pouch using a linear probe with in plane approach. (Fig. 2) An ultrasound guided approach is used as it can guarantee injection into the joint space [46]. The injection procedures will be conducted under aseptic technique [47]. If both knees are painful, only the more painful knee will be injected. Injections will be conducted at week $0,4,8$ and 16 for both groups. In case of pain flares after injection, the subsequent injection will be commenced after the flare is subsided, or at 1 month. If participants display allergic symptoms to the injected solution, therapy will be terminated but participants will continue to be followed in their allocated group until the end of the study.

Participants in the intervention group will receive injections with loaded syringe containing $5 \mathrm{ml} 25 \%$ dextrose (D25), prepared by mixing $2.5 \mathrm{ml} \mathrm{50 \%} \mathrm{dextrose}$ with $2.5 \mathrm{ml}$ sterile Water for Injection BP. D25 is a commonly used concentration for intra-articular DPT injection and has been used in previous studies [21, 22]. Participants in the control group will receive injections of $5 \mathrm{ml} \mathrm{NS}$. Recent level one evidence has suggested that NS yields a statistically and clinically meaningful improvement in KOA-related pain up to 6 months after the injection. Therefore, NS will serve as the active control in this trial [48].

Post-injection care: Participants will be observed for 10 min post-intervention consistent with clinical practice and studies [21, 22]. Participants will be advised to take only acetaminophen (500 to $1000 \mathrm{mg}$ every 4 to $6 \mathrm{~h}$ as-needed) and avoid non-steroidal antiinflammatory drugs in the first $48 \mathrm{~h}$ after injection, 


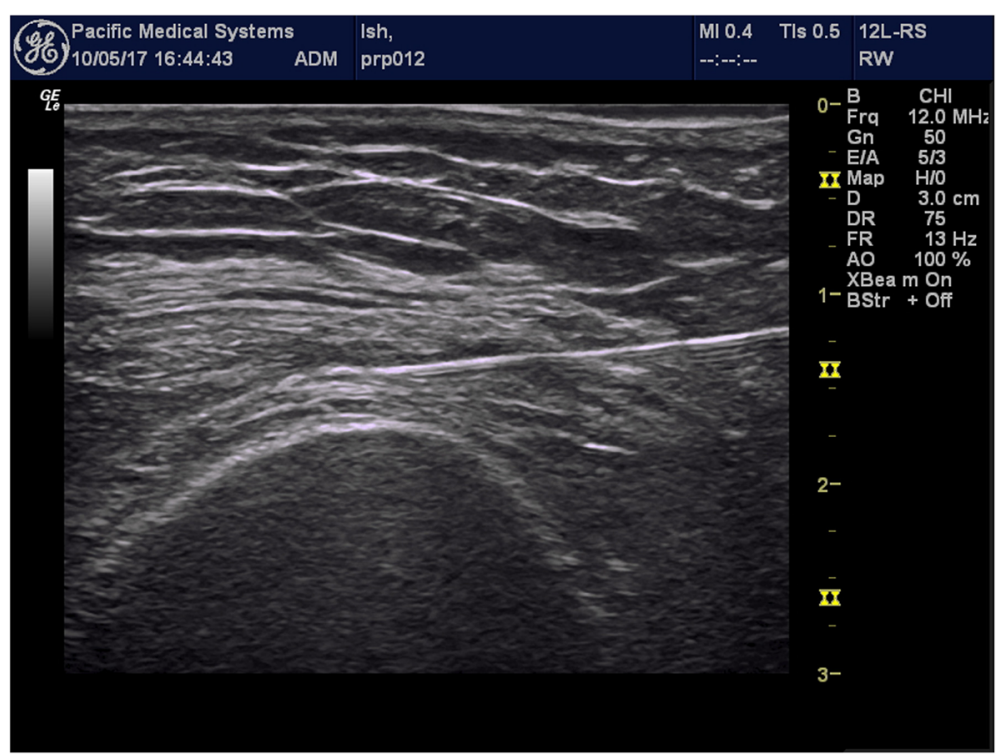

Fig. 2 Suprapetella pouch injection under USG guidance

which may interfere with the DPT mechanism of action. They will be allowed to take their usual analgesics medication after $48 \mathrm{~h}$. Participants will be instructed on post-injection care and slow ramp-up of activity. Participants will be contacted 2 days after the injection to assess for side effects.

A home-based post-treatment quadriceps strengthening exercise will be prescribed to both groups and participants will be encouraged to practice daily. Cointerventions will be allowed to both groups, such as conventional medication, physical therapy, acupuncture, herbal medicine, over-the-counter drugs and other active treatments. The use of co-interventions will be retrieved from the Clinical Management System (CMS), an electronic system operated by the Hospital Authority in Hong Kong. Participants will be asked to recall their private treatment as well. However, participants are restricted to receive other injection therapies during the study period.

\section{Outcome measurement \\ Primary outcome}

The Western Ontario McMaster University Osteoarthritis Index (WOMAC) pain score is the primary outcome. WOMAC is a disease-specific quality of life questionnaire for use in osteoarthritis clinical trials [33]. It consists of 24 self-reported items including knee pain (5 items), stiffness (2 items) and function (17 items). The subscales fulfill conventional criteria for content and construct validity, reliability, responsiveness and relative efficiency [49].

\section{Secondary outcomes}

Secondary outcomes include WOMAC composite score, the WOMAC function and stiffness score. Objective physical function is measured using the 30-s chair stand performance test, $40 \mathrm{~m}$ fast-paced walk test and the timed up -and-go test (TUGT). The three tests are recommended by the Osteoarthritis Research Society International (OARSI) as performance-based tests to assess physical function in people diagnosed with KOA [50]. Health related quality of life is assessed using the EuroQuol-5 questionnaire. The measure has strong construct validity, responsiveness and clinometric profile; it has been used to assess the economic impact of OA [35]. Joint pain will also be assessed by the Visual Analogue Score (VAS) in $0-100 \mathrm{~mm}$ scale upon walking in the past $48 \mathrm{~h}$. Analgesic consumption will be assessed by the number of participants on analgesics by the 7-day recall diary. Before un-blinding, treatment satisfaction will be tested by asking "Would you recommend the therapy to others with knee OA like yours?"

\section{Data collection and management}

Data will be collected at baseline, and at week 16 , 26 and 52 . We will record the number of potential candidates, responses received and their resolution, and the number of injection and assessment sessions attended. Follow up data will include the number at each follow up, the number of participants completing the trial, and the number of withdrawals due to all causes. Data entry, transfer and subsequent maintenance will be performed by a data manager. An 
electronic database will be used, and the server is in a physically secured location with backup on weekly basis. Access to study data is restricted to the study research team by username and password.

\section{Safety monitoring}

Participants have a diary to document any discomfort after each injection; they are advised to call to the study coordinator if they are uncertain whether the discomfort is related to the injections. Standardization forms will be used for monitoring and reporting of side effects and adverse events. The principal investigator will be present in case of a significant adverse event. The principal investigator will report serious adverse events to the ethics committee within $24 \mathrm{~h}$, and annual reports summarizing adverse events will be submitted to the Drug Office of the Department of Health, Hong Kong Administrative Region.

\section{Statistical issues Sample size Calculation}

The sample size calculation is based on 2 RCTs which evaluated the intra-articular injection of DPT and NS for $\mathrm{KOA}$ at 6 months $[32,51]$, assuming their therapeutic effect will remain stable for 1 year. The baseline characteristics of the two trials had comparable age, sex, BMI, and baseline WOMAC score. The mean (Standard Deviation) difference of WOMAC scores at 6 month compared with baseline status was 25.2 (20.3) points for DPT [32], and 9.53 (26.6) points for NS [51], respectively. Thus, assuming a pooled SD of 26.6, a sample size of 34 participants in each arm will have $80 \%$ power to detect a significant effect size of 0.70 in a two-sample ttest with alpha set at 0.05 . Assuming $10 \%$ dropout rate, the total sample size is 76 .

\section{Data analysis}

We will conduct linear mixed models to investigate significant changes over time for both primary and secondary outcomes following the intention to treat principle, i. e., all available data will be analyzed according to the group they are randomly assigned. The use of LMM also provides the means to include subjects with incomplete and use all available data to assess the treatment effect over time. In this model, intervention group, time, and the interactions between the intervention groups and time will serve as predictors with duration of knee pain and number of comorbidities as covariates. Given the longitudinal nature of the clinical trial data, we assume the autoregressive covariance structure will be the best fit for the data, but the statistical fitness by using other covariance structures will also be evaluated [52].

With a clearly defined target population, effectiveness and safety outcomes, and convenient data collection procedures, our trial should realize the goal of maximizing the number of participants who are maintained on the protocol-specified intervention until the outcome data are collected. In case of a large number of outcomes and covariates with missing data, we will use multivariate imputation using chained equations (MICE) to incorporate auxiliary information about the missing data. The imputation model will include prerequisite variables in the data analysis, variables for baseline socio-economic status, and also variables that are predictors of outcomes. About 10 iterations will be conducted in each imputation process with more iterations to be considered until the chain reaches convergence [53]. Twenty completed datasets will be imputed with the use of the chain equations. The Rubin's rule will be applied to combine the effect estimates [54]. This approach provides estimated standard errors and $P$ values that incorporate missing-data uncertainty. For the patients that meet any of the exclusion criteria such as alternative treatment or severe outcome, then all subsequent longitudinal measurements since that date of the event will be excluded from the analysis.

\section{Discussion}

One of the objectives of International Association for the Study of Pain 2016 is to encourage research aimed at producing more effective and accessible treatment methods and outcomes for people with joint pain. Intra-articular injections remain a popular non-surgical treatment options for KOA. The current injection therapies include corticosteroids, hyaluronic acid and platelet rich plasma. Intra-articular corticosteroid is well known for its short-term pain relief up to 4 weeks only, and is usually indicated for acute inflammatory flares. Intra-articular hyaluronic acid appears to have longer pain relief up to 6 months, though its cost-effectiveness remains controversial. Intra-articular platelet rich plasma is emerging rapidly in recent years, but high quality scientific evidence of efficacy is lacking. In spite of the growing evidence of efficacy and effectiveness of DPT, it appears to be under-utilized in the medical world. Thus, there is a compelling need to conduct a high quality RCT with rigorous study design to determine the clinical efficacy of a brief intervention, IA DPT for KOA. IA DPT is safe, easily accessible and inexpensive; the injection can be easily performed by trained physicians. The trial follows the recommendations from the OARSI, and evaluates both validated self-reported and objective functional outcomes, as well as quality-oflife. Results will translate directly to clinical practice. Statistically and clinically positive outcomes on IA DPT would provide immediate, practical benefits to 
individual patients and society at-large through improved quality-of-life and decrease use of medical resources. Since dextrose is inexpensive, there is potential economic impact to the healthcare system in the management of KOA. Positive results from this high quality trial would suggest IA DPT could be used as non-surgical treatment for KOA.

\begin{abstract}
Abbreviations
ANCOVA: Analysis of covariance; ANOVA: Analysis of variance; BMI: Body mass index; CONSORT: CONsolidated standards of reporting trials; CREC: Clinical research ethics committee; D25: $5 \mathrm{ml}$ 25\% dextrose; DPT: Dextrose prolotherapy; EQ-5D: EuroQol-5D; GOPCs: General outpatients clinics; IPAQ: International physical activity questionnaire; KOA: Knee osteoarthritis; LMM: Linear mixed models; NS: Normal saline; NTE: New Territories East; OARSI: Osteoarthritis research society international; RCT: Randomized controlled trial; SNOSE: Sequentially numbered, opaque sealed envelopes; TCM: Traditional Chinese medicine; TKR: Total knee replacement; TUGT: Timed up -and-go test; VAS: Visual analogue score; WOMAC: Western Ontario McMaster University Osteoarthritis Index
\end{abstract}

\section{Funding}

The study is funded by the Chinese University of Hong Kong Direct Grant for Research 2013-14 (HKD 40,000). The funding body has no role in the study other than provide funding.

\section{Availability of data and materials}

The datasets used and/or analysed during the current study are available from the corresponding author on reasonable request.

\section{Authors' contributions}

RWSS is the principal investigator of the study, responsible for the conception and study design, literature review and writing of the protocol. She will continue to coordinate the trial and responsible for data interpretation and completion of the final manuscript. RWKW is responsible to conduct the knee injections. KDR, DR and VCHC contribute to the conception and study design, writing up of the protocol, and they will continue their support in data interpretation and completion of the manuscript. DCCC and BHKY are responsible for sample size calculation and statistical analytical plan. They will be responsible for future data analysis and contribute on writing the statistical results in the final manuscript. SYSW contributes to the study design and writing of the protocol, and he will continue his support in data interpretation and completion of the manuscript. All authors read and approved the final manuscript.

\section{Ethics approval and consent to participate}

The study complies with the Declaration of Helsinki, and has been approved by the Joint Chinese University of Hong Kong - New Territories East Cluster Clinical Research Ethics Committee. (CREC no. 2014.059). Written inform consent was obtained from all participants.

\section{Competing interests}

The authors declare that they have no competing interests.

\section{Publisher's Note}

Springer Nature remains neutral with regard to jurisdictional claims in published maps and institutional affiliations.

\section{Author details}

'Jockey Club School of Public Health and Primary Care, The Chinese University of Hong Kong, New Territories, Hong Kong. ${ }^{2}$ Department of Physical Medicine and Rehabilitation (1986-2015), The University of Kansas, Kansas City, USA. ${ }^{3}$ Department of Family Medicine, University of Wisconsin School of Medicine and Public Health, Madison, WI, USA.
Received: 20 June 2017 Accepted: 2 May 2018

Published online: 15 May 2018

\section{References}

1. Felson DT. Osteoarthritis of the knee. N Engl J Med. 2006;354(8):841-8.

2. Felson DT. Epidemiology of osteoarthritis. In: Brandt KDDM, Lohmander LS, editors. Osteoarthritis. Oxford, England: Oxford university press; 2003. p. 9-16.

3. Woo J, Leung J, Lau E. Prevalence and correlates of musculoskeletal pain in Chinese elderly and the impact on 4-year physical function and quality of life. Public Health. 2009;123(8):549-56.

4. Lawrence RC, Felson DT, Helmick CG, et al. Estimates of the prevalence of arthritis and other rheumatic conditions in the United States: part II. Arthritis \& Rheumatism. 2008:58(1):26-35.

5. Gupta S, Hawker GA, Laporte A, Croxford R, Coyte PC. The economic burden of disabling hip and knee osteoarthritis $(\mathrm{OA})$ from the perspective of individuals living with this condition. Rheumatology (Oxford, England). 2005;44(12):1531-7.

6. Sayer EC, Li LC, Kopec JA, Esdaile JM, Cibere J. The effect of disease site (knee, hip, hand, foot, lower back or neck) on employment due to osteoarthritis. PLoS One 2010;DOl: https://doi.org/10.1371/journal.pone.0010470.

7. Hutton I, Gamble G, Mclean G, Butcher H, Gow P, Dalbeth N. Obstacles to action in arthritis: a community case-control study. Int J Rheum Dis. 2009;12(2):107-17.

8. Samson DJ, Grant MD, Ratko TA, Bonnell CJ, Ziegler KM, Aronson N. Treatment of primary and secondary osteoarthritis of the knee. Evid. Rep. Technol. Assess. 2007;157:1-157.

9. Altman RD. Recommendations for the medical management of osteoarthritis of the hip and knee: 2000 update. American College of Rheumatology Subcommittee on Osteoarthritis Guidelines. Arthritis Rheum. 2000:43:1905-15.

10. Little CV, Parsons T. Herbal therapy for treating osteoarthritis. The Cochrane database of systematic reviews. 2001;1:Cd002947.

11. Morelli V, Naquin C, Weaver V. Alternative therapies for traditional disease states: osteoarthritis. Am Fam Physician. 2003;67(2):339-44.

12. Carr AJ, Robertsson O, Graves S, et al. Knee replacement. Lancet (London, England). 2012;379(9823):1331-40.

13. Felson DT. Osteoarthritis: priorities for osteoarthritis research: much to be done. Nature Rev Rheumatol. 2014;10(8):447.

14. Conaghan PG, Kloppenburg M, Schett G, Bijlsma JW. Osteoarthritis research priorities: a report from a EULAR ad hoc expert committee. Annals of the rheumatic diseases. 2014:73(8):1442-5.

15. Felson DT. The sources of pain in knee osteoarthritis. Curr Opin Rheumatol. 2005:17(5):624-8.

16. Hawker GA, Gignac MA, Badley E, et al. A longitudinal study to explain the pain-depression link in older adults with osteoarthritis. Arthritis Care \& Res. 2011;63(10):1382-90.

17. Hochman J, Davis A, Elkayam J, Gagliese L, Hawker G. Neuropathic pain symptoms on the modified painDETECT correlate with signs of central sensitization in knee osteoarthritis. Osteoarthr Cartil. 2013;21(9):1236-42.

18. Rabago D, Slattengren A, Zgierska A. Prolotherapy in primary care. Prim Care. 2010;37:65-80

19. Reeves KD, Sit RW, Rabago DP. Dextrose Prolotherapy: A Narrative Review Of basic science, clinical research, and best treatment recommendations. Phys Med Rehabil Clin N Am. 2016;27(4):783-823.

20. Maniquis-Smigel L, Reeves KD, Lyftogt J, Rabago D. Analgesic Effect of Caudal 5\% Dextrose in Water in Chronic Low Back Pain (Abs). Arch. Phys. Med. Rehabil. 2015;96(10):e103

21. Rabago D, Zgierska A, Fortney L, et al. Hypertonic dextrose injections (Prolotherapy) for knee osteoarthritis: results of a single-arm uncontrolled study with 1-year follow-up. J Altern Complement Med. 2012;18(4):408-14.

22. Rabago D, Patterson JJ, Mundt M, et al. Dextrose prolotherapy for knee osteoarthritis: a randomized controlled trial. Ann Fam Med. 2013;11(3):229-37.

23. Dumais $R$, Benoit C, Dumais $A$, et al. Effect of regenerative injection therapy on function and pain in patients with knee osteoarthritis: a randomized crossover study. Pain Med. 2012:13(8):990-9.

24. Hassan F, Trebinjac S, Murrell WD, Maffulli N. The effectiveness of prolotherapy in treating knee osteoarthritis in adults: a systematic review. Br Med Bull. 2017:1-18.

25. Nourani B, Rabago D. Prolotherapy for knee osteoarthritis: a descriptive review. Current Physical Medicine and Rehabilitation Reports. 2016;4(1):42-9.

26. Sit RW, Chung VC, Reeves KD, Rabago D, Chan KK, Chan DC, Wu X, Ho RS, Wong SY. Hypertonic dextrose injections (prolotherapy) in the treatment of 
symptomatic knee osteoarthritis: a systematic review and meta-analysis. Sci Rep. 2016;6:25247.

27. Hung C-Y, Hsiao M-Y, Chang K-V, Han D-S, Wang T-G. Comparative effectiveness of dextrose prolotherapy versus control injections and exercise in the management of osteoarthritis pain: a systematic review and metaanalysis. J Pain Res. 2016;9:847-57.

28. Reeves KD, Hassanein K. Randomized prospective double-blind placebocontrolled study of dextrose prolotherapy for knee osteoarthritis with or without ACL laxity. Altern Ther Health Med. 2000;6(2):68.

29. Eslamian F, Amouzandeh B. Therapeutic effects of prolotherapy with intraarticular dextrose injection in patients with moderate knee osteoarthritis: a single-arm study with 6 months follow up. Ther Adv Musculoskelet Dis. 2015;7(2):35-44.

30. Topol GA, Podesta LA, Reeves KD, Giraldo MM, Johnson LL, Grasso R, Jamín A, Clark T, Rabago D. Chondrogenic effect of intra-articular hypertonic-dextrose (prolotherapy) in severe knee osteoarthritis. PM\&R. 2016;8(11):1072-82.

31. Hashemi SM, Madadi F, Razavi S, Nikooseresht M, Nasiripour S. Intra-articular hyaluronic acid injections Vs. dextrose prolotherapy in the treatment of osteoarthritic knee pain. Tehran University of Medical Sciences. 2012;70(2).

32. Hashemi M, Jalili P, Mennati $S$, et al. The effects of prolotherapy with hypertonic dextrose versus prolozone (intraarticular ozone) in patients with knee osteoarthritis. Anesth Pain Med. 2015;5(5).

33. McAlindon TE, Driban JB, Henrotin $Y$, et al. OARSI clinical trials recommendations: design, conduct, and reporting of clinical trials for knee osteoarthritis. Osteoarthr Cartil. 2015;23(5):747-60.

34. Dobson F, Hinman RS, Roos EM, et al. Recommended performance-based tests to assess physcial function in people diagnosed with hip or knee osteoarthritis. Osteoarthr Cartil. 2013;21(18):1042-52.

35. Fransen $\mathrm{M}$, Edmonds J. Reliability and validity of the EuroQol in patients with osteoarthritis of the knee. Rheumatology. 1999;38(9):807-13.

36. Chan A-W, Tetzlaff JM, Altman DG, et al. SPIRIT 2013 statement: defining standard protocol items for clinical trials. Ann Intern Med. 2013;158(3):200-7.

37. Aigner T, Rose J, Martin J, Buckwalter J. Aging theories of primary osteoarthritis: from epidemiology to molecular biology. Rejuvenation Res. 2004;7(2):134-45.

38. Altman RD. Criteria for classification of clinical osteoarthritis. J Rheumatol Suppl. 1991;27:10-2.

39. Randolph TG, Rollins JP, Walter CK. Allergic reactions following the intravenous injection of corn sugar (dextrose). Arch Surg. 1950;61(3):554-64.

40. Guharoy S, Barajas M. Probable anaphylactic reaction to corn-derived dextrose solution. Vet Hum Toxicol. 1991;33(6):609-10.

41. Kernan WN, Viscoli CM, Makuch RW, Brass LM, Horwitz RI. Stratified randomization for clinical trials. J Clin Epidemiol. 1999;52(1):19-26.

42. Schulz KF, Grimes DA. Allocation concealment in randomised trials: defending against deciphering. Lancet. 2002:359(9306):614-8.

43. Bang H, Ni L, Davis CE. Assessment of blinding in clinical trials. Control Clin Trials. 2004:25(2):143-56.

44. Qu NN, Li KJ. Study on the reliability and validity of international physical activity questionnaire (Chinese Vision, IPAQ). Zhonghua liu xing bing xue za zhi = Zhonghua liuxingbingxue zazhi. 2004;25(3):265-8.

45. Kellgren JH, Lawrence JS. Radiological assessment of osteo-arthrosis. Ann Rheum Dis. 1957;16(4):494-502

46. Maricar N, Parkes MJ, Callaghan MJ, Felson DT, O'Neill TW. Where and how to inject the knee—a systematic review. Semin Arthritis Rheum. 2013;43(2):195-203.

47. Hart S. Using an aseptic technique to reduce the risk of infection. Nurs Stand. 2007;21(47):43-8.

48. Saltzman BM, Leroux T, Meyer MA, et al. The therapeutic effect of intraarticular normal saline injections for knee osteoarthritis: a meta-analysis of evidence level 1 studies. Am J Sports Med. 2017:45(11):2647-53.

49. Bellamy N, Buchanan WW, Goldsmith CH, Campbell J, Stitt LW. Validation study of WOMAC: a health status instrument for measuring clinically important patient relevant outcomes to antirheumatic drug therapy in patients with osteoarthritis of the hip or knee. J Rheumatol. 1988;15(12):1833-40.

50. Dobson F, Hinman RS, Hall M, Terwee CB, Roos EM, Bennell KL. Measurement properties of performance-based measures to assess physical function in hip and knee osteoarthritis: a systematic review. Osteoarthr Cartil. 2012;20(12):1548-62.

51. Yang KA, Raijmakers N, Van Arkel E, et al. Autologous interleukin-1 receptor antagonist improves function and symptoms in osteoarthritis when compared to placebo in a prospective randomized controlled trial. Osteoarthr Cartil. 2008;16(4):498-505.
52. Akaike H. Likelihood of a model and information criteria. J Econ. 1981; 16(1):3-14.

53. Van Buuren S. Multiple imputation of discrete and continuous data by fully conditional specification. Stat Methods Med Res. 2007;16(3):219-42.

54. Rubin DB. Multiple imputation for nonresponse in surveys. Vol 81: john Wiley \& sons; 2004.

\section{Ready to submit your research? Choose BMC and benefit from:}

- fast, convenient online submission

- thorough peer review by experienced researchers in your field

- rapid publication on acceptance

- support for research data, including large and complex data types

- gold Open Access which fosters wider collaboration and increased citations

- maximum visibility for your research: over $100 \mathrm{M}$ website views per year

At BMC, research is always in progress.

Learn more biomedcentral.com/submissions 